\title{
Improved Detection of Helicobacter pylori Infection and Premalignant Gastric Mucosa Using Conventional White Light Source Gastroscopy
}

\author{
Likit Matrakool $^{1}$, Taweesak Tongtawee ${ }^{1 *}$, Theeraya Bartpho', Chavaboon \\ Dechsukhum $^{2}$, Ryan A Loyd ${ }^{3}$, Soraya J Kaewpitoon ${ }^{3}$, Natthawut Kaewpitoon ${ }^{4}$
}

\begin{abstract}
Background: The gold standard diagnosis of $H$. pylori related gastritis is evidence of bacteria on histopathological examination of gastric mucosa. Our aim was to study the correlation between gastric mucosal morphology and histopathological severity of $\boldsymbol{H}$. pylori related gastritis. Materials and Methods: Division was made on morphological features into: Type 1, showing regular arrangement of red dots; Type 2, showing cleft-like appearance; Type 3, with a mosaic appearance; and Type 4, having a mosaic appearance with focal or diffuse hyperemia. Results: Types 1 and 2 gastric mucosal morphologies were statistically significant in predicting an $H$. pylori negative status $(137 / 145, p<0.01)$, while Types 3 and 4 were significant a positive status $(139 / 155, p<0.01)$. The sensitivity, specificity, positive and negative predictive values of Type 3 and 4 morphologies for predicting H.pylori positive were $94.6 \%, 89.5 \%, 89.7 \%$ and $94.5 \%$, respectively, with a good correlation with inflammation grading $(\mathbf{p}<0.01)$. Conclusions: Our study suggests that gastric mucosal morphology can be reliably identified using conventional white light source gastroscopy with good correlation between findings and inflammation grading.
\end{abstract}

Keywords: Gastric mucosa - premalignant gastric lesion - conventional white light source gastroscopy - H pylori

Asian Pac J Cancer Prev, 17 (4), 2099-2103

\section{Introduction}

Gastroscopy and biopsy for the rapid urease test and histopathological examination have been still standard diagnosis for $H$. pylori infection. Many studies have been attempted to classify the gastroscopic findings of $H$.pylori related gastritis. In 2002, Japanese endoscopist found that collecting venules, seen as numerous minute red dots in the gastric corpus, where a characteristic finding in the normal gastric mucosa morphology without $H$. pylori infection using both standard and magnifying endoscopy (Yagi et al., 2002). This finding was termed "regular arrangement of collecting venules" (RAC).

In 2005, Japanese endoscopists provided more precise information concerning the network of collecting venules (Yagi et al., 2005). The gastroscopic findings of H.pylori infected gastric mucosa were erythema, erosions, antral nodularity, thickened gastric folds, and visible submucosal vessels. However, these findings are not a reliable method of diagnosis because of their low sensitivity and specificity (Laine et al., 1995; Bah et al., 1995; Mihara et al., 1999; Redeen $\mathrm{S}$ et al., 2003). In 2010, Taiwanese endoscopist a study using close-up observation between the endoscope tip and the gastric mucosa and found the "mosaic pattern" in the corpus mucosa. This study can improve accuracy of H. pylori infection status (Yan et al., 2010). They classified gastric mucosal morphology into two categories (normal RAC and abnormal mosaic pattern). However, the classification was insufficient to predict all $H$. pylori infections.

In our study, we used 4 categories; Type1: showing regular arrangement of red dots (RAC), Type2: showing cleft-like appearance, Type 3: mosaic appearance, Type 4 mosaic appearance with focal or diffuse hyperemia. One recent study from the United States has indicated the usefulness of NBI for predicting $H$. pylori infection and the occurrence of intestinal metaplasia in the stomach (Anagnostopoulos et al., 2007). Many reports suggest that high resolution magnification endoscopy has been proved in the identification of normal gastric mucosa and H. pylori-related gastritis (Yagi et al., 2002). Our recent study, gastric mucosal morphological patterns in the $H$. pylori infected gastric mucosa can be reliably identified using C-NBI gastroscopy with good correlation

${ }^{1}$ School of Surgery, ${ }^{2}$ School of Pathology, ${ }^{3}$ School of Family Medicine and Community Medicine, Institute of Medicine, Suranaree University of Technology, ${ }^{4}$ Faculty of Public health, Vongchavalitkul University, Nakhon Ratchasima, Thailand $*$ For correspondence: taweesak.t@sut.ac.th 
with inflammation grading (Taweesak et al., 2015). The endoscopist can improve accuracy of gastric biopsy using the site specific biopsy technique (Taweesak et al., 2015). The Kyoto global consensus report on H. pylori gastritis suggested that atrophic mucosa and intestinal metaplasia can be accurately detected by image enhanced endoscopy, after appropriate training (Kentaro et al., 2015).

However, practicing high resolution magnification endoscopy in daily gastroscopic examination seems not to be feasible, because it takes more examination time and needs more experience of the endoscopist. If specific mucosal morphology of $H$.pylori-related gastritis can be identified using conventional white light source gastroscopy, they may be applicable to targeted biopsy of the suspected area of $H$. pylori infection in daily clinical practice. The aim of our study to classify the gastric mucosal morphology of $H$. pylori related gastritis using conventional white light source gastroscopy and correlation with severity grading of gastritis according to Sydney classification (Dixon et al., 1994; Tytgat et al., 1991).

\section{Materials and Methods}

\section{Patients}

A 200 patients underwent gastroscopy for the investigation of dyspeptic symptoms were enrolled in the our study from January 2014 to November 2014 in the Endoscopy unit, Suranaree Medical Center, Department of Surgery, Institute of Medicine, Suranaree University of Technology, Nakhon Ratchasima, Thailand. The following exclusion criteria were applied: age below 18 or above 70 years, $H$. pylori eradication treatment in the previous 2 months, suspected or confirmed malignancy on endoscopy, significant medical illnesses and history of previous gastric surgery, the use of antimicrobials or gastrointestinal medications like PPIs, H2blockers or bismuth compounds within the previous 2 months. All patients provided informed consent, and the study was approved by the institutional review board of Suranaree University of Technology, Nakhon Ratchasima, Thailand.

\section{Diagnosis of H. pylori infection}

Adiagnosis of $H$.pylori infection was made if $H$.pylori bacteria were seen on histopathological examination and the rapid urease test was positive. Patients with negative results in one or both examinations were considered to be H.pylori negative, according to the European guidelines for the diagnosis of $H$. pylori infection (Malfertheiner $\mathrm{P}$ et al., 2002).

\section{Biopsy specimens}

Four biopsy samples were taken directly from the observation area as shown in Figure 1. Two samples were sent for histological analysis and two were used for rapid urease testing on site (ProntodyleR, GASTREX, France)

\section{Histological analysis}

Specimens for histological analysis were placed in $10 \%$ formalin solution and routinely processed. The hematoxylin and eosin stain and Giemsa stain were used for identification of $H$. pylori. All of the cases were evaluated by 5 pathologists of Bangkok Pathological Laboratory outside Suranaree University.

\section{Endoscopic findings}

The local anesthesia was the same as for conventional white light source gastroscopy. The gastroscopic procedures were performed using an upper GI videoendoscope (Olympus EVIS EXERA III, CV-190) in all cases. The whole stomach was examined with conventional white light source gastroscopy. The gastric mucosa was chosen for observation. The observed gastric mucosal morphology was classified into 4 morphologies. Type: 1 showing the regular arrangement of collecting venules, type 2: mucosal pattern showing cleft like appearance, Type 3: showing the mosaic appearance, Type 4 showing the mosaic appearance with focal or diffuse hyperemia.

\section{Image evaluation}

All gastroscopic examinations were digitally recorded and still images of the observation sites were captured for use in the reproducibility study. The selected images were transferred to a software program without distorting brightness, contrast or color balance. An endoscopist classified them as type 1 through type 4 gastric mucosal morphology as described above. All endoscopists were blinded to the results of the H.pylori status and histology before reviewing the slides.

\section{Inter and intraobserver agreement study}

During endoscopy, close-up still images were captured in the gastric antrum and the body of the stomach. Among the high quality images showing clear gastric mucosal morphology, a total of 200 pictures from 200 patients were selected for the intra and interobserver agreement study. All endoscopists were blinded to the results of the $H$. pylori status and histology before reviewing the pictures.

\section{Statistical analysis}

The sensitivity, specificity, and positive and negative predictive values of the various gastric mucosal morphologies were calculated. Clinicopathologic factors associated with the accuracy of predicting $H$. pylori positivity were assessed by logistic regression analysis. A p-value of $<0.05$ was considered significant. All statistical analyses were performed using the SPSS, version 16.0 (SPSS Inc., Chicago, IL, USA). The $x$ value was calculated for inter and intra observer variabilities. $\mathrm{k}$ values below 0.4 indicated poor agreement, values between 0.4 and 0.8 represent moderate agreement, values between 0.6 and 0.8 represented substantial agreement, and values greater than 0.8 corresponded to excellent agreement.

\section{Results}

A total of 200 consecutive patients (92 men, 118 women; mean age 49.0 years, range 19-69 years) were enrolled in our study from January 2014 to November 2014. The 200 patients included: 35 patients showing a type 1 pattern, 25 patients showing a type 2 pattern, 60 patients showing a type 3 pattern, 80 patients showing 
Improved Detection of Helicobacter pylori Infection and Premalignant Gastric Mucosa Using White Light Source Gastroscopy

a type 4 pattern (Table 1). H. pylori infection was demonstrated by both a positive result of the rapid urease test as well as bacteria seen on histological examination in 132 patients (66\%). Type 1 and type 2 gastric mucosal morphology were statistically significant in predicting $H$. pylori negative status as compared with other mucosal morphologies $(58 / 68, \mathrm{p}<0.01)$. Type 3 and type 4 gastric mucosal morphology was statistically significant in predicting $H$. pylori positive status as compared with other mucosal morphologies $(130 / 140, \mathrm{p}<0.01)$. Furthermore, the sensitivity, specificity, positive and negative predictive values of type 3 and type 4 morphologies for predicting $H$. pylori positive were $98.48 \%, 92.85 \%, 98.48 \%$, and $94.48 \%$ respectively with good correlation with inflammation grading according to the Sydney classification $(\mathrm{p}<0.01)$.

Gastric mucosal morphology and severity of gastric mucosal inflammation

Type 1 showing the regular arrangement of collecting venules, gastric mucosal morphology was associated with a regular arrangement of surface epithelium, with absent or minimal infiltration by inflammatory cells (Figure 1). Type 2 abnormal gastric mucosal morphology corresponded to mild gastritis with mild glandular atrophy, mild infiltration by inflammatory cells, irregular arrangement of surface epithelium, and irregular opening pits (Figure 2). Moderate gastritis was recognized in type 3 , with moderate glandular atrophy, moderate infiltration by inflammatory cells, and irregular arrangement of surface epithelium (Figure 3).

Different conventional white light source gastroscopy of gastric mucosal morphologies and correlation with

Table 1. Correlation between Gastric Mucosal Morphology and $\boldsymbol{H}$. pylori Infection Status

\begin{tabular}{cccc}
\hline & \multicolumn{2}{c}{ H. pylori infection status } & \\
\cline { 2 - 3 } $\begin{array}{c}\text { Mucosal } \\
\text { morphology }\end{array}$ & $\begin{array}{c}\text { Non-infected } \\
\text { subjects } \\
(\mathrm{HP}-)\end{array}$ & $\begin{array}{c}\text { infected } \\
\text { subjects } \\
(\mathrm{HP}+)\end{array}$ & p-value \\
& $35(35 / 35)$ & - & $<0.01$ \\
Type 1 & $23(23 / 25)$ & $2(2 / 25)$ & $<0.01$ \\
Type2 & $5(5 / 60)$ & $55(55 / 60)$ & $<0.01$ \\
Type3 & $5(5 / 80)$ & $75(75 / 80)$ & $<0.01$ \\
Type4 & & & \\
\hline
\end{tabular}

Table 2. Correlation between Gastric Mucosal Morphology and Inflammation Grading

\begin{tabular}{|c|c|c|c|c|}
\hline \multirow{2}{*}{$\begin{array}{c}\text { Mucosal } \\
\text { morphology }\end{array}$} & \multicolumn{3}{|c|}{ Inflammation grading } & \multirow[t]{2}{*}{ p-value } \\
\hline & mild & moderate & severe & \\
\hline Type 1 & $35(35 / 35)$ & - & - & $<0.01$ \\
\hline Type2 & $10(10 / 25)$ & $15(15 / 25)$ & - & $<0.01$ \\
\hline Type3 & $5(5 / 60)$ & $55(55 / 60)$ & - & $<0.01$ \\
\hline Type4 & - & $25(25 / 80)$ & $\begin{array}{c}55 \\
(55 / 80)\end{array}$ & $<0.01$ \\
\hline
\end{tabular}

histopathological severity

Marked gastritis was found in type 4, with marked glandular atrophy, marked lymphocytic infiltration, lymphoid follicular hyperplasia, and intestinal metaplasia (Figure 4).

Interobserver and intraobserver agreement assessment

The k-values for inter and intra observer agreement for the gastroscopic mucosal morphologies were significant. The k-values for inter and intra observer agreement with regard to prediction of $H$. pylori infection status were also significant (Table 3).

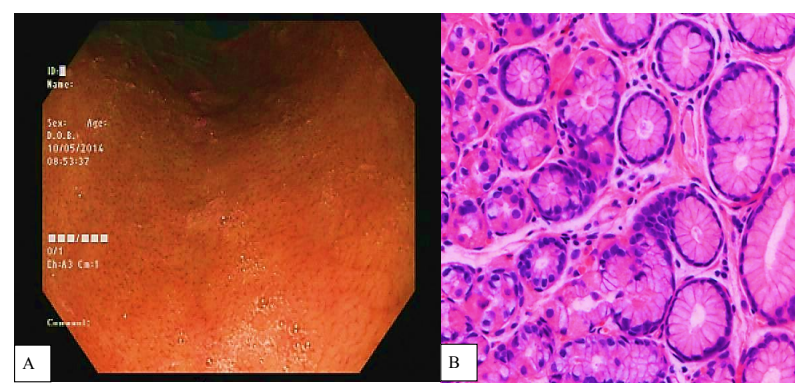

Figure 1. Type 1 Gastric Mucosal Morphology. Note regular arrangement of collecting venules (A) and associated with regular arrangement of surface epithelium, with absent or minimal infiltration by inflammatory cells (B)

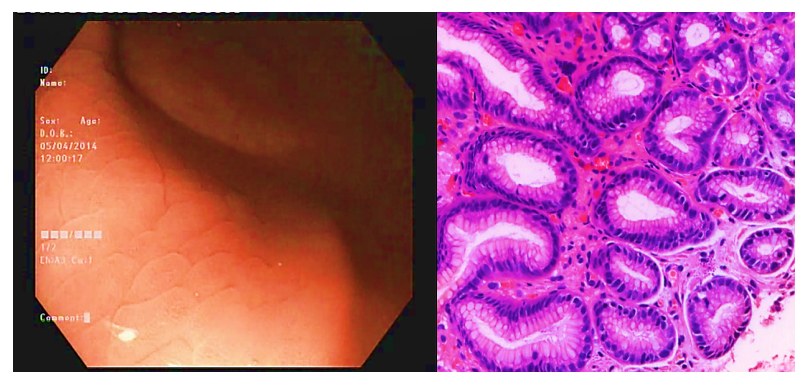

Figure 2. Type 2 Gastric Mucosal Morphology. Note cleft-like appearance (A) and associated mild infiltration by inflammatory cells, irregular arrangement of surface epithelium, and irregular opening pits (B)

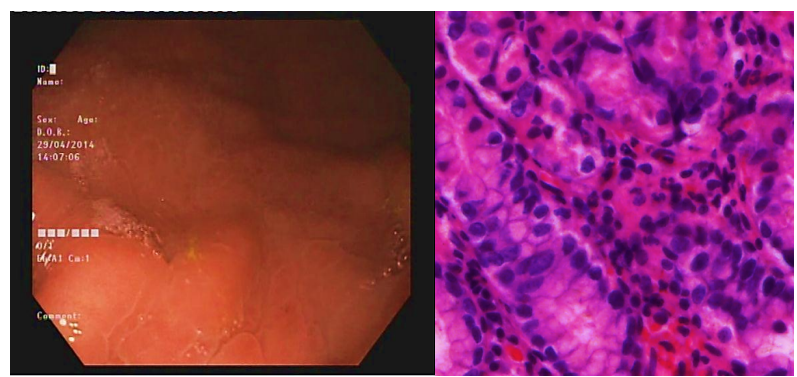

Figure 3. Type 3 Gastric Mucosal Morphology. Note mosaic appearance (A) and associated moderated infiltration by inflammatory cells (B)

Table 3. Inter- and Intra-Observer Agreement

\begin{tabular}{ccccc}
\hline & \multicolumn{2}{c}{ Interobserver agreement } & \multicolumn{2}{c}{ Intraobserver agreement } \\
\cline { 2 - 6 } & $\%$ agreement & k value $(95 \% \mathrm{CI})$ & $\%$ agreement & $\mathrm{k}$ value $(95 \% \mathrm{CI})$ \\
\hline Gastric mucosal morphology & 91.8 & $0.91(0.88-0.92)$ & 89.9 & $0.88(0.85-0.90)$ \\
H. pylori infection status & 96.9 & $0.98(0.96-0.97)$ & 98.3 & $0.97(0.97-0.98)$ \\
\hline
\end{tabular}

The k-values for inter- and intraobserver agreement for the various gastric mucosal morphologies were significant. CI, confidence interval 


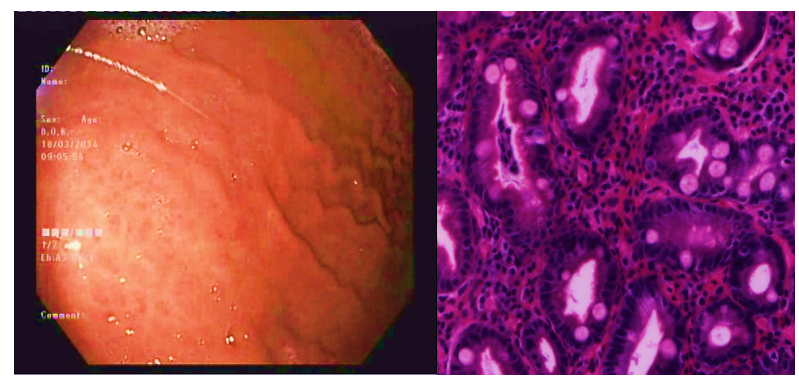

Figure 4. Type 4 Gastric Mucosal Morphology. Note mosaic appearance with focal area of hyperemia (A) and associated marked infiltration by inflammatory cell and present of intestinal metaplasia (B)
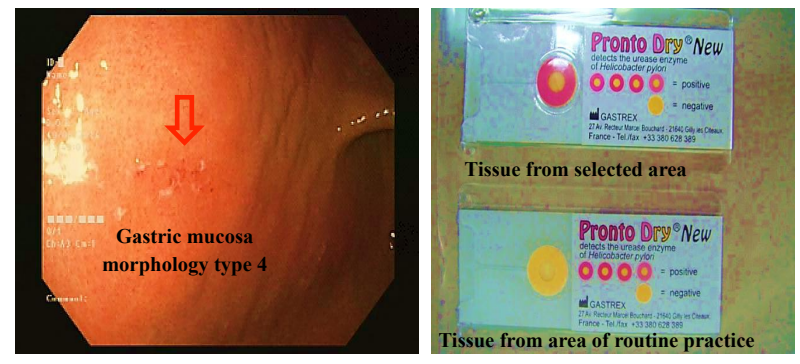

Figure 5. Gastric Mucosal Morphology Guided Biopsy

\section{Discussion}

Evident of bacteria on histopathological examination is therefore still considered to be the gold standard for diagnosis $H$. pylori related gastritis. The reliability of detecting H.pylori related gastritis and other conditions such as atrophy and intestinal metaplasia by "blind" biopsy sampling of gastric mucosa depend on the site, number, and size of biopsy specimens. This practice could result in sampling errors, missed pathology, and unnecessary work and costs for pathology departments.

Early diagnosis and eradication of $H$. pylori is a key step in eliminating gastric malignancy. Real time identification of the site of $H$. pylori related gastritis in the stomach during gastroscopy not only reduces the sampling error and excessive workload of pathologist, but also improves detection of early malignant lesions by indicating the need for more meticulous examination of the whole stomach. The development of high resolution magnified endoscopy markedly overcame these problems. Yet the use of magnified imaging for routine daily screening for $H$. pylori related gastritis is impossible in routine practice. It is not only costly, but also less widely available, and is also time consuming. In addition, it needs special patient preparation and a well-trained endoscopist. In our study, the gastric mucosal morphology was classified into 4 types using conventional white light source gastroscopy.

Gastric mucosal morphologies type 1 and type 2 were statistically significant in predicting $H$. pylori negative status as compared with other mucosal morphologies $(58 / 68, \mathrm{p}<0.01)$. Type 3 , type 4 gastric mucosal morphology was statistically significant in predicting $H$. pylori positive status as compared with other mucosal morphologies $(130 / 140, \mathrm{p}<0.01)$. Furthermore, the sensitivity, specificity, positive and negative predictive values of type 3, type 4 morphologies for predicting $H$. pylori positive were $98.48 \%, 92.85 \%, 98.48 \%$, and $94.48 \%$ respectively with good correlation with inflammation grading according to the Sydney classification $(\mathrm{p}<0.01)$.

In conclusion, considering the unsatisfactory sensitivity of conventional biopsy for diagnosing $H$. pylori and the limitations of the magnified image, our study suggests that gastric mucosal morphology in $H$. pylori infected gastric mucosa can be reliably identified using conventional white light source gastroscopy and can predict the histopathological severity of gastritis. In this study suggests that gastric mucosal morphology in the $H$. pylori infected gastric mucosa can be reliably identified using conventional white light source gastroscopy with good correlation with inflammation grading. Findings from our studies have shown a good correlation between gastric mucosal morphology and H. pylori status and severity of pathological inflammation grading especially premalignant gastric lesion. This benefit for target areas of the biopsy with suspected $H$. pylori infection in daily practice (Figure 5). Further studies to compare between gold standard biopsy and gastric mucosal morphology guided biopsy.

\section{Acknowledgements}

This study was supported by a grant for medical investigation from Suranaree University of Technology and was approved by the Ethics Committee for Research Involving Human Subjects Suranaree University of Technology (EC-57-34).

\section{References}

Anagnostopoulos GK, Yao K, Kaye P, et al (2007). Highresolution magnification endoscopy can reliably identify normal gastric mucosa, Helicobacter pylori associated gastritis, and gastric atrophy. Endoscopy, 39, 202-7.

Bah A, Saraga E, Armstrong D, et al (1995). Endoscopic features of Helicobacter pylori-related gastritis. Endoscopy, 27, 593-6.

Dixon MF, Genta RM, Yardley JH, et al (1996). Classification and grading of gastritis. The updated Sydney System. International Workshop on the Histopathology of Gastritis, Houston. Am J Surg Pathol, 20, 1161-81.

Laine L, Cohen H, Sloane R, et al (1995). Interobserver agreement and predictive value of endoscopic findings for H. pylori and gastritis in normal volunteers. Gastrointest. Endosc, 42, 420-3.

Malfertheiner P, Mégraud F, O'Morain C, et al (2002). Current concepts in the management of Helicobacter pylori infection-the Maastricht 2-2000 Consensus Report. Aliment Pharmacol Ther, 16, 167-80.

Mihara M, Haruma K, Kamada T, et al (1999). The role of endoscopic findings for the diagnosis of Helicobacter pylori infection: evaluation in a country with high prevalence of atrophic gastritis. Helicobacter, 4, 40-8.

Redeen S, Petersson F, Jonsson KA, et al (2003). Relationship of gastroscopic features to histological findings in gastritis and Helicobacter pylori infection in a general population sample. Endoscopy, 35, 946-50.

Sugano K, Tack J, Kuipers EJ, et al (2015). Kyoto global 
Improved Detection of Helicobacter pylori Infection and Premalignant Gastric Mucosa Using White Light Source Gastroscopy consensus report on Helicobacter pylori gastritis. Gut, 64, 1353-67.

Taweesak T, Soraya K, Natthawut K, et al (2015). Correlation between gastric mucosal morphologic patterns and histopathological severity of Helicobacter pylori associated gastritis using conventional narrow band imaging gastroscopy. Biomed Res Int, 7, 42-8.

Taweesak T, Chavaboon D, Wilairat L, et al (2015). Improved detection of Helicobacter pylori infection and premalignant gastric mucosa using "site specific biopsy": a randomized control clinical Trial. Asian Pac J Cancer Prev, 16, 8487- 90.

Tytgat GN (1991). The Sydney System: endoscopic division. Endoscopic appearances in gastritis/duodinitis. $J$ Gastroenterol Hepatol, 6, 223-34.

Yagi K, Nakamura A, Sekine A, et al (2002). Characteristic endoscopic and magnifiedendoscopic findings in the normal stomach without Helicobacter pylori infection. J. Gastroenterol. Hepatol, 17, 39-45.

Yagi K, Nakamura A, Sekine A, et al (2002). Comparison between magnifying endoscopy and histological, culture and urease test findings from the gastric mucosa of the corpus. Endoscopy, 34, 376-81.

Yagi K, Honda H, Yang JM, et al (2005). Magnifying endoscopy in gastritis of the corpus. Endoscopy, 37, 660-6.

Yan SL, Wu ST, Chen CH et al (2010). Mucosal patterns of Helicobacter pylori-related gastritis without atrophy in the gastric corpus using standard endoscopy. World $J$ Gastroenterol, 16, 496-500. 